Research Article

\title{
Preparation of sulfur hydrophobized plasmonic photocatalyst towards durable superhydrophobic coating material
}

\author{
Emese Lantos a ${ }^{\mathrm{a}}$ László Mérai ${ }^{\mathrm{a}}$, Ágota Deák ${ }^{\mathrm{a}}$, Juan Gómez- Pérez ${ }^{\mathrm{b}}$, Dániel Sebők ${ }^{\mathrm{a}}$, \\ Imre Dékány ${ }^{\mathrm{a}}$, Zoltán Kónya ${ }^{\mathrm{b}}$, László Janovák ${ }^{\mathrm{a}, *}$ \\ a University of Szeged, Interdisciplinary Excellence Centre, Department of Physical Chemistry and Materials Science, H-6720, Rerrich Béla tér 1, Szeged, \\ Hungary \\ b University of Szeged, Interdisciplinary Excellence Centre, Department of Applied and Environmental Chemistry, H-6720, Rerrich Béla tér 1, Szeged, Hungary
}

\section{A R T I C L E I N F O}

\section{Article history:}

Received 6 February 2019

Received in revised form 23 March 2019

Accepted 15 April 2019

Available online 16 November 2019

\section{Keywords:}

Nanocomposite

Coating materials

Wetting

Lotus-type structure

Visible light photocatalyst

\begin{abstract}
A B S T R A C T
The widely used photocatalytic self-cleaning coating materials are often made of polymers and polymer based composites, where the photocatalyst immobilization occurs with macromolecules. However, these organic polymers are often unstable under exposure to UV irradiation and easily degraded by reactive radicals produced in the photocatalytic reaction. In order to solve this problem, in this paper, we present the facile preparation of a multifunctional coating with dual superhydrophobic and photocatalytic properties, where the fixation and the hydrophobization of the plasmonic $\mathrm{Ag}-\mathrm{TiO}_{2}$ photocatalyst particles with visible light activity was performed with non-water soluble sulfur, which is a cheap and easily available material. The resulted novel nanocomposite with rough and nano-tructured surface roughness (1.25-2.45 nm determined by small-angle X-ray scattering) has sufficient low surface energy $\left(3.3 \mathrm{~mJ} / \mathrm{m}^{2}\right)$ for superhydrophobic $\left(\theta=151.1^{\circ}\right)$ properties. Moreover, in contrast of the organic and expensive fluoropolymer based composites, this non-wetting nature was durable, because the measured $\theta$ was higher than $150^{\circ}$ during the long- term LED $\left(\lambda_{\max }=405 \mathrm{~nm}\right)$ light irradiation.
\end{abstract}

(c) 2019 Published by Elsevier Ltd on behalf of The editorial office of Journal of Materials Science \& Technology.

\section{Introduction}

Photocatalyst-based functional surfaces are one of the state-ofthe-art materials that are commercially applied in several fields, including wastewater and air treatment or even food-packing. After the discovery of the photo-induced superhydrophilicity of photocatalyst (e.g., $\mathrm{TiO}_{2}$ ) layers [1], commercially applicable photofunctional materials with self-cleaning properties were synthesized using $\mathrm{TiO}_{2}$ particles and composite thin films. Since the band gap of $\mathrm{TiO}_{2}$ falls within the UV-A range, it is possible to extend the absorption spectrum of the semiconductor photocatalysts by modifying the catalyst particles with, for example, different metallic or non-metallic elements [2-4]. The functionalized catalysts can be excited by the UV-A or visible (VIS) light due to the surface plasmon resonance effect: the metal nanoparticles absorb electromagnetic radiation at lower energy wavelengths [4,5].

The incorporation of semiconductor photocatalyst particles in an appropriate polymer-ased support or binder material is expected to create antimicrobial and self-cleaning properties,

\footnotetext{
* Corresponding author.

E-mail address: janovakl@chem.u-szeged.hu (L. Janovák).
}

which would extend its field of application. The state-of-the-art materials at the area of photocatalyst/polymer composite layers are the functional surfaces, which are gaining attention for different practical fields of application, such as food packing materials with antibacterial behaviours [6] and self-cleaning superhydrophobic coating $[7,8]$.

Since their discovery, superhydrophobic surfaces $\left(\theta \geq 150^{\circ}\right.$, low contact angle hysteresis) are in focus owing to their low surface free energy and beneficial self-cleaning nature. Besides the originally hydrophobic nature of the material, a surface has to be textured in both the micro-, and nano-scale to possess superhydrophobic characteristics [9]. There are numerous reported ways to produce morphologically proper surfaces, including lithographic, chemical etching, templates and even spray-coating methods, which is cheap, scaleable, and easily implementable. In this case, particulate material (e.g., a photocatalyst) gets sprayed on a substrate, forming the desired surficial structures with adequate surface roughness. If hydrophobized photocatalysts are used as roughness enhancer particulate materials, it results in the formation of bifunctional composite surfaces with dual photocatalytic and extreme wetting properties [10-13].

When polymer immobilized photoreactive hybrid surfaces are prepared, the photodegradation of the matrix also has to be taken 
into consideration, so the reportedly applied hydrophobic organic polymers (e.g. perfluorynated polyacrylates, or PTFE) may be inappropriate for long term uses. Because of this, the demand is high for durable matrix materials. To satisfy the needs for increased durability, sulfur also can be applied as an inert, non-photodegradable and insoluble inorganic hydrophobizing agent. There are various application of elemental sulfur and sulfur nanoparticles nowadays such as agrochemical industries [14] fungicides in agriculture fields [15], modification of carbon nano-tubes [16], antibacterial material [17], and synthesis of nano composites for lithium batteries [18]. However, according to our best of knowledge, this is the first demonstration of utilizing sulfur nanoparticles as inert hydrophobizing agent for creation of superhydrophobic and photoreactive functional surface.

Thus, in this paper, we present a simple and cheap spraycoating method to prepare non-photodegradable VIS light-active and micro/nanostructured superhydrophobic surfaces on inorganic sulfur basis: the VIS light-activity owes to the $10 \mathrm{wt} \%$ plasmonic Ag$\mathrm{TiO}_{2}$ photocatayst content, while the durable superhydrophobic nature is attributed to sulfur-nanoparticles, prepared by a simple precipitation method in aqueous medium. These bifunctional inorganic composite layers with long-term durability showed considerable photocatalytic efficiency during $\mathrm{EtOH}$ (g) degradation tests at solid/ gas interface.

\section{Materials and methods}

\subsection{Preparation of the sulfur hydropobized $\mathrm{Ag}-\mathrm{TiO}_{2}$ layers}

The VIS light-active plasmonic $\mathrm{Ag}-\mathrm{TiO}_{2}$ photocatalyst with $0.5 \mathrm{wt} \%$ surface silver nanoparticle content was synthesized via the direct functionalization of $\mathrm{TiO}_{2}$ particles. The synthetic method and the optical characterization of the catalyst are detailed in our previous publication $[4,5]$.

To obtain sulfur nanoparticles, $0.03 \mathrm{~g}$ of sulfur powder (Sigma Aldrich) was dissolved in $4 \mathrm{ml}$ of $10 \mathrm{M}$ aqueous $\mathrm{NaOH}$-solution $(\mathrm{pH}=14.15)$ at a temperature of $80^{\circ} \mathrm{C}$. Afterwards, the liquid was stirred then diluted with $1 \mathrm{ml}$ of $10 \mathrm{M} \mathrm{NaOH}$ solution, then $4.65 \mathrm{ml}$ of $2.5 \mathrm{M} \mathrm{H}_{2} \mathrm{SO}_{4}$ solution was added drop wise until the dispersion became turbid ( $\mathrm{pH}=13.95$ at precipitation) due to the formed sulfur nanoparticles $(c \approx 0.09 \mathrm{~g} / 100 \mathrm{ml})$.

The resulting precipitated sulfur was centrifuged $(8000 \mathrm{rpm}$, $5 \mathrm{~min}$ ), thoroughly washed with distilled water and dried overnight in a drying cabinet at $60^{\circ} \mathrm{C}$. The mixing of the obtained sulfur nanoparticles and the previously synthetized $\mathrm{Ag}-\mathrm{TiO}_{2}$ photocatalyst occurred in aqueous dispersion $(c=0.01 \mathrm{~g} / 100 \mathrm{ml})$. The sulfur/ photocatalyst ratio $(0,40,60,80,85,90$ and $100 \mathrm{wt} \% \mathrm{~S}$-content) was systematically changed during the preparation.

To prepare $5 \mathrm{~cm} \times 5 \mathrm{~cm}$ photoreactive inorganic layers consisting of $10 \mathrm{wt} \% \mathrm{Ag}-\mathrm{TiO}_{2}$ photocatalyst and $90 \mathrm{wt} \%$ sulfur nanoparticles, the aqueous dispersions were evenly sprayed on glass substrates, using an R180-type airbrush spray gun at an operating pressure of 3 bar.

\subsection{Methods of sample characterization}

The size distribution and morphology of the prepared sulfur, and the $\mathrm{Ag}-\mathrm{TiO}_{2} / \mathrm{S}$ composite nanoparticles were characterized during high resolution transmission electron microscopy (HRTEM). The applied FEI Tecnai G2 20 X-TWIN microscope, is equipped with a tungsten cathode ( $200 \mathrm{kV}$ acceleration voltage).

Surface morphology of the $\mathrm{Ag}-\mathrm{TiO}_{2} / \mathrm{S}$ layers was examined with a scanning electron microscope (SEM, Hitachi S-4700, secondary electron detector, acceleration voltage: 10 or $20 \mathrm{kV}$ ), while the surficial composition was studied using a Röntec EDS detector.
Optical characterization by diffuse reflectance UV-VIS spectra were recorded via using a CHEM2000 UV-VIS (USB2000+UV-VIS, Ocean Optics Inc., Dunedin, FL) spectrophotometer, equipped with an integrated sphere.

The surface fractal dimensions $\left(D_{\mathrm{S}}\right)$ and surface roughness (SR) of the samples were investigated by small-angle X-ray scattering (SAXS) technique. SAXS curves were recorded with a slit collimated Kratky compact small-angle system (KCEC/3 Anton-Paar KG, Graz, Austria) equipped with a position-sensitive detector (PSD $50 \mathrm{M}$ from M. Braun AG. Munich, Germany) containing 1024 channels, $54 \mu \mathrm{m}$ in width. $\mathrm{CuK \alpha}$ radiation was generated by using a Philips PW1830 X-ray generator operating at $40 \mathrm{kV}$ and $30 \mathrm{~mA}$. The Kratky camera was calibrated using silver behenate with a well-defined lamellar structure $(d=5.848 \mathrm{~nm})$.

Surface charge values of the photocatalyst and the sulfur suspensions were measured by means of a particle charge detector (PCD-04 Particle Charge Detector; Mütek Analytic GmbH, Germany) with manual titration. Under a titration process the positive surface charge of the $\mathrm{Ag}-\mathrm{TiO}_{2}$ photocatalysts particles (in 0.01 \% aqueous medium, $\mathrm{pH}=5.0$ set by $\mathrm{HCl}$ ) will be compensated with negatively charged sulfur nanoparticles (in $0.09 \%$ aqueous medium, $\mathrm{pH}=5.0$ set by $\mathrm{HCl}$ ) with concomitant streaming potential measurements.

Composition-dependent crystalline properties were studied using a Philips powder X-ray diffractometer (PW 1830 generator, PW 1820 goniometer, $\mathrm{Cu}_{\alpha}: \lambda=0.1542 \mathrm{~nm}, 40-50 \mathrm{kV}, 30-40 \mathrm{~mA}$, $2 \theta: 2^{\circ}-70^{\circ}, 25.0 \pm 0.5^{\circ} \mathrm{C}$ ).

The Raman spectra were recorded with $1.5 \mathrm{~cm}^{-1}$ interferometric resolution applying a Senterra (Bruker) Raman microscope under $50 \mathrm{x}$ magnification with $2.50 \mathrm{~mW}$ output power of the $\lambda=532 \mathrm{~nm}$ He-Ne laser light source and $3000 \mathrm{~ms}$ integration time. The presented spectra are the coadditions of 5 single spectra in all cases, recorded at different spots of the sample.

The apparent static contact angles on $\mathrm{Ag}-\mathrm{TiO}_{2} / \mathrm{S}$ layers were measured according to the sessile drop technique at $25.0 \pm 0.5^{\circ} \mathrm{C}$ under atmospheric pressure, applying an EasyDrop drop shape analysis system (Krüss GmbH, Hamburg, Germany) equipped with DSA100 software, a Peltier temperature chamber and a steel syringe needle of $0.5 \mathrm{~mm}$ diameter, and using distilled water as a test liquid.

Beside the static contact angles the dynamic wetting of the composites was also measured because according to the theory of Drelich and Chibowski $[13,19]$ the measured advancing $\left(\theta_{\text {adv }}\right)$ and receding $\left(\theta_{\text {rec }}\right)$ contact angles are suitable for the estimation of the total apparent surface free energy $\left(\gamma_{\mathrm{s}}{ }^{\text {tot }}\right)$ of the layer, knowing the surface tension of the probe liquid, $\left(\gamma_{1}=72.1 \mathrm{mN} / \mathrm{m}\right.$ in the case of distilled water at $25^{\circ} \mathrm{C}$ ) and its contact angle hysteresis, which is defined as the difference between the $\theta_{\mathrm{adv}}$ and $\theta_{\mathrm{rec}}$, as shown by Eq. (1):

$\gamma_{\mathrm{s}}^{\mathrm{tot}}=\frac{\gamma_{1}\left(1+\cos \theta_{\mathrm{adv}}\right)^{2}}{2+\cos \theta_{\mathrm{rec}}+\cos \theta_{\mathrm{adv}}}$

To study the photocatalytic properties of the $\mathrm{Ag}-\mathrm{TiO}_{2} / \mathrm{S}$ layers, ethanol (EtOH) degradation tests were run under blue LED light (General Electric's, Veresegyház, Hungary, $\lambda=405 \mathrm{~nm}$ ) illumination at solid/gas interface, while gas chromatographic measurements were carried out (Shimadzu GC-14B) to quantify the rate of EtOH degradation. During the measurements, $5 \mu$ l of EtOH (abs., Molac Chemicals, Hungary) was injected into the reaction chamber containing $25 \mathrm{~cm}^{2}\left(25.0 \pm 0.5^{\circ} \mathrm{C}\right)$ hybrid layers $\left(5 \mathrm{mg} / \mathrm{cm}^{2}\right)$, fixed at a $5 \mathrm{~cm}$ distance from the light source. The $\mathrm{c} / \mathrm{c}_{0}$ values calculated from peak areas were determined as the function of illumination time, where $c$ is the concentration of EtOH at time $(t)$ and $c_{0}$ is the initial concentration $\left(c_{0}=0.36 \mathrm{mM}\right)$. The apparent reaction rate constants $\left(k^{\prime}, \min ^{-1}\right)$ were calculated for first-order decay as shown by the equation: $\ln \left(c / c_{0}\right)=-k^{\prime} t$. 


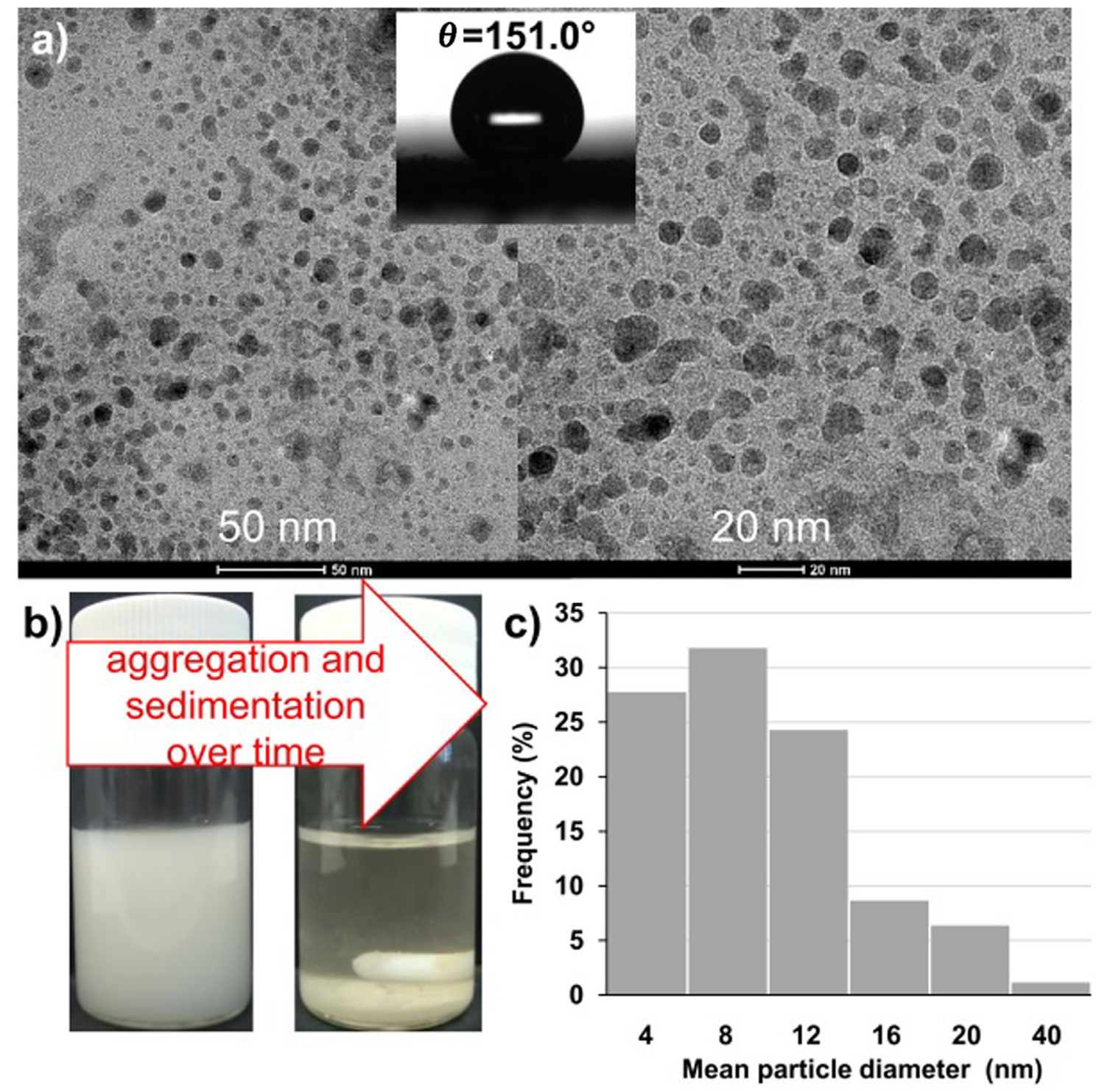

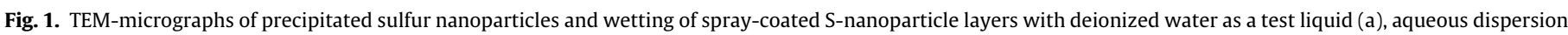
of prepared sulfur nanoparticles upon precipitation and $24 \mathrm{~h}$ later (b), size-distribution of precipitated sulfur nanoparticles (c).

During the stability tests, photoreactive composite layers of $5 \mathrm{mg} / \mathrm{cm}^{2}$ specific mass were illuminated for 14 days by the same blue LED-light $(\lambda=405 \mathrm{~nm}$ ) placed at a $5 \mathrm{~cm}$ distance from the samples, under ambient atmosphere. The changes in water contact angle of the layers was measured daily during the experiment. Polymer based composite thin films with 60 wt\% incorporated $\mathrm{Ag}-\mathrm{TiO}_{2}$ particles were used as reference.

Similar to previous work [4], the adhesive tape test was used to investigate the mechanical properties of the prepared composite films. Briefly, the double-sided adhesive foam tape ( $1.6 \mathrm{~mm}$ thick) was adhered to the films firstly, and a $100 \mathrm{~g}$ weight was rolled over the tape to ensure consistency. The tape was then peeled back at an angle of approximately $45^{\circ}$.

\section{Results and discussion}

\subsection{Structural characterization of composite nanoparticles}

According to the Wenzel and Cassie-Baxter models [20], to reach the superhydrophobic extreme wetting character, the provision of hierarchical roughness to a naturally hydrophobic surface is crucial.
To achieve this goal, sulfur nanoparticles with a maximum diameter of $40 \mathrm{~nm}$ were synthesized by applying a simple precipitation method. Hence there are many reported bottom-up ways to prepare sulfur nanoparticles, including the sulfur vapour deposition on cold water [21] or the Weimarn- [22], and Raffo-methods [23], only the last one offers particle yields higher enough $(\sim 600 \mathrm{~g} / \mathrm{l})$ in aqueous media to be industrially profitable [24]. Our version of the applied Raffo-method is based on the following chemical reactions [25]:

$$
\begin{aligned}
& \mathrm{S}+6 \mathrm{NaOH}=\mathrm{Na}_{2} \mathrm{~S}_{2} \mathrm{O}_{3}+2 \mathrm{Na}_{2} \mathrm{~S}+3 \mathrm{H}_{2} \mathrm{O} \\
& \mathrm{Na}_{2} \mathrm{~S}_{2} \mathrm{O}_{3}+\mathrm{H}_{2} \mathrm{SO}_{4}=\mathrm{H}_{2} \mathrm{~S}_{2} \mathrm{O}_{3}+\mathrm{Na}_{2} \mathrm{SO}_{4} \\
& x \mathrm{H}_{2} \mathrm{~S}_{2} \mathrm{O}_{3}=y \mathrm{H}_{2} \mathrm{O}+z \mathrm{SO}_{2}+\mathrm{H}_{2} \mathrm{~S}_{m} \mathrm{O}_{6} \\
& \mathrm{H}_{2} \mathrm{~S}_{m} \mathrm{O}_{6}=\mathrm{H}_{2} \mathrm{~S}_{m-n} \mathrm{O}_{6}+\mathrm{S}_{n}(n>5)
\end{aligned}
$$

Upon the acidification of $\mathrm{S}_{2} \mathrm{O}_{3}{ }^{2-}$-containing solutions with sulfuric acid, $\mathrm{SO}_{2}$ and colloidal $\mathrm{S}$ forms. We obtained $\mathrm{S}_{2} \mathrm{O}_{3}{ }^{2-}$ containing solution as a result of dissolving elemental $\mathrm{S}$ in $\mathrm{pH}=14.15 \mathrm{NaOH}$-solution at $80^{\circ} \mathrm{C}$, then $\mathrm{S}$-nanoparticles with a maximum mean diameter of $40 \mathrm{~nm}$ formed upon the following addition of $2.5 \mathrm{M} \mathrm{H}_{2} \mathrm{SO}_{4}$ solution, which is the core step of the 



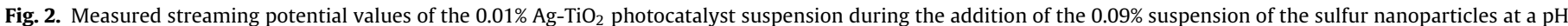

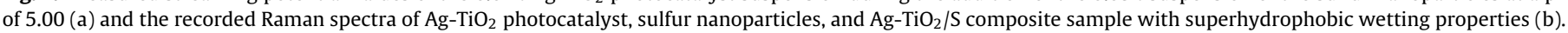

classical Raffo-method. According to Eqs. (2-5), the dissolution of sulfur in such media is a disproportionation reaction, while the reverse precipitation reaction is synproportionation. As our process requires both elemental and processed $\mathrm{S}\left(\mathrm{H}_{2} \mathrm{SO}_{4}\right)$ and offers good yields its industrial application could be beneficial in the elimination of residual $\mathrm{S}$ as a by-product of the petrol industry.

As shown in Fig. 1(a), the size distribution of the obtained spherical sulfur nanoparticles is relatively polydisperse. The mean diameters range between 1 and $40 \mathrm{~nm}$, which is comparable to the size of the applied VIS light-active plasmonic $\mathrm{Ag}-\mathrm{TiO}_{2}$ photocatalyst particles $\left(d_{\text {prim. }} \sim 25 \mathrm{~nm}\right)[4,5]$. Because the hydrophobic nature of the sulfur particles, the stability of the obtained aqueous suspension was very low.

The sulfur nanoparticles also show superhydrophobic character $\left(\theta=151.0^{\circ}\right)$ as they are evenly dispersed on plain surfaces (Fig. 1(b)), because the formed sulfur layer consists of hydrophobic nonwater-soluble nanoparticles with rough surface in the nanometer scale. The fixation of the plasmonic $\mathrm{Ag}-\mathrm{TiO}_{2}$ photocatalyst particles on the surface of the sulfur particles was executed through electrostatic interaction because the $\mathrm{pH}$-dependent surface charge of $\mathrm{TiO}_{2}$ and $\mathrm{Ag}-\mathrm{TiO}_{2}$ is well-known in the literature and it has positively charged below $\mathrm{pH}=6$ (point of zero charge) [25], while the charge of the sulfur nanoparticles was slightly negative (point of zero charge at $\mathrm{pH}=2$ [24]). Thus, the surface fixation was carried out at $\mathrm{pH}=5$ because at this value the components have opposite charge and the obtained charge titration curve achieved at this $\mathrm{pH}$ is presented on Fig. 2(a). It can be seen that the positive charge of the $\mathrm{Ag}-\mathrm{TiO}_{2}$ particles was continuously decreased during the addition of the negatively charged sulfur suspension. The inserted photos also show that at the charge compensation point (streaming potential $=0$ ) the stability of the initial $\mathrm{Ag}-\mathrm{TiO}_{2}$ and sulfur suspension was drastically decreased because of the lost (i.e., compensated) charge of the components and the formation of the heterocoagulated $\mathrm{S} / \mathrm{Ag}-\mathrm{TiO}_{2}$ system.

Fig. 2(b) shows the recorded Raman spectra of or $\mathrm{S}, \mathrm{Ag}-\mathrm{TiO}_{2}$, and $\mathrm{Ag}-\mathrm{TiO}_{2} / \mathrm{S}$ composite nanoparticles. Hence the $\mathrm{P} 25 \mathrm{TiO}_{2}$ used during the synthesis of the plasmonic photocatalyst consist of $75 \mathrm{wt} \%$ anatase and $25 \mathrm{wt} \%$ rutile, only the characteristic peaks of anatase at $144 \mathrm{~cm}^{-1}\left(E_{\mathrm{g}}\right), 397.5 \mathrm{~cm}^{-1}\left(B_{1 \mathrm{~g}}\right), 517.5 \mathrm{~cm}^{-1}\left(B_{1 \mathrm{~g}}\right)$ and $637.5 \mathrm{~cm}^{-1}$
( $\left.E_{\mathrm{g}}\right)$ could be detected upon $\lambda=532 \mathrm{~nm}$ laser-light illumination [26].

The presence of silver is undetectable at these experimental conditions, due to its low concentration ( $0.5 \mathrm{wt} \%)$. In the case of sulfur samples, peaks (with the corresponding molecular vibrational modes) at $82.5 \mathrm{~cm}^{-1}\left(B_{2 \mathrm{~g}}\right), 153 \mathrm{~cm}^{-1}\left(E_{\mathrm{g}}\right), 187.5 \mathrm{~cm}^{-1}\left(E_{\mathrm{u}}\right)$, $219 \mathrm{~cm}^{-1}\left(A_{1 \mathrm{~g}}\right), 244.5 \mathrm{~cm}^{-1}\left(A_{1 \mathrm{~g}}\right), 435 \mathrm{~cm}^{-1}\left(B_{2 \mathrm{~g}}\right)$ and $472.5 \mathrm{~cm}^{-1}$ $\left(A_{1 \mathrm{~g}}\right.$ and $\left.E_{\mathrm{g}}\right)$ are characteristics to the vibrational bonds of $\mathrm{S}_{8^{-}}$ molecules $[27,28]$, while the peak at $51 \mathrm{~cm}^{-1}$ belongs to the $a_{\mathrm{g}}$ lattice-vibrational mode. Fig. 2(b) also shows, that if we form superhydrophobic composite from the initial sulfur, and $\mathrm{Ag}-\mathrm{TiO}_{2}$ nanoparticles, the intensity of sulfur peaks increases, while at $90 \mathrm{wt} \% \mathrm{~S}$-content, the characteristic peaks of rutile almost disappear, indicating the decreased surface concentration of the photocatalyst. However, no chemical interaction was identified between the sulpur and the $\mathrm{Ag}-\mathrm{TiO}_{2}$ particles evidenced by Raman measurements.

Fig. 3 shows the powder X-ray diffractograms of the resulting composites: as the sulfur-content increases, the characteristic peak of the anatase structure of $\mathrm{TiO}_{2}$ at $2 \theta=25.32^{\circ}$ decrease in intensity, while other peaks appear, attributed to the orthorhombic $\alpha$-sulfur (with higher intensities: $23.10^{\circ}, 25.88^{\circ}, 26.76^{\circ}, 27.76^{\circ}, 28.96^{\circ}$ ) [29], making the identification of the photocatalyst more difficult due to convolution.

Moreover, some peaks are characteristics to monoclinic $\beta$-sulfur $\left(27.74^{\circ}, 31.08^{\circ}, 37.84^{\circ}\right)$ [30]. The observations indicate the crystalline nature of the precipitated sulfur-nanoparticles, since no broad band of amorphous phase were detected. As can be seen in the inserted pictures of Fig. 3, the two components powder samples have high dispersibility in aqueous media under $90 \mathrm{wt} \%$ sulfurcontent, but this water dispersibility drastically drops at higher sulfur contents. This indicates an optimally hydrophobic nature and low surface free energy to be applied in surface hydrophobization.

Beside the above presented X-ray diffractograms results, the composites were also characterized by small-angle X-ray scattering (SAXS) method because the fractal plot of these scattering curves gives reliable information about the quality of the surface roughness (SR) with a quantitative information based on the surface fractal dimensions. The double logarithmic (fractal) plot of 


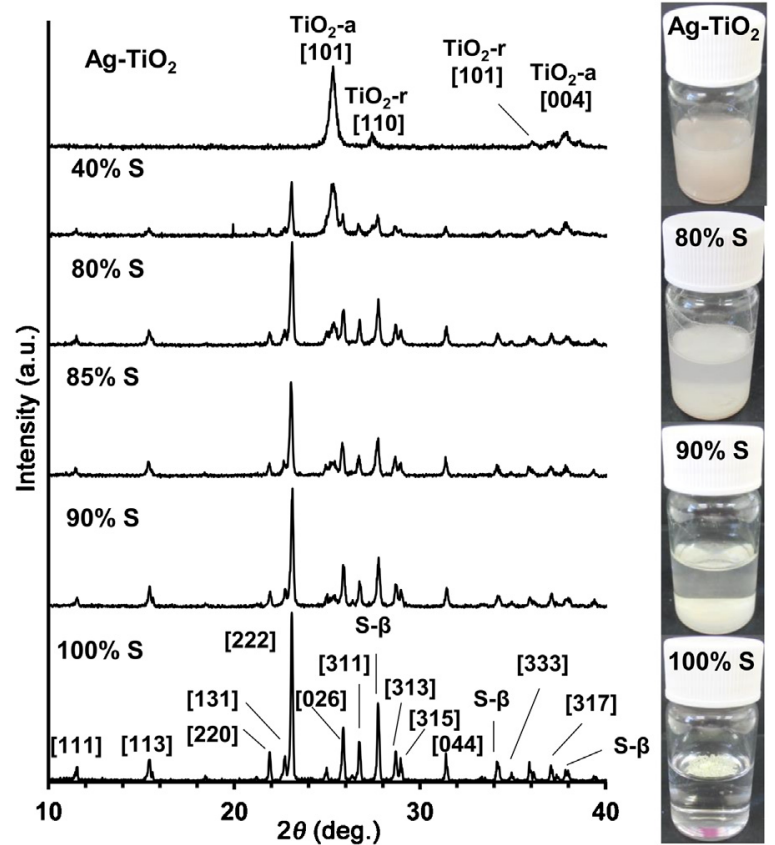

Fig. 3. Powder X-ray diffractograms and aqueous dispersions of $\mathrm{Ag}-\mathrm{TiO}_{2}, \mathrm{~S}$ and $\mathrm{Ag}$ $\mathrm{TiO}_{2} / \mathrm{S}$.

the scattering curves of $\mathrm{Ag}-\mathrm{TiO}_{2}$ and $\mathrm{Ag}-\mathrm{TiO}_{2} / \mathrm{S}$ powder samples with $60 \mathrm{wt} \% \mathrm{~S}$-content shows that the samples have a rather rough surface, indicated by the $D_{\mathrm{S}}=2.8$ (Fig. 4(a)).

Furthermore, the $h^{3} I(h)$ vs. $h^{2}$ plot of the curves shows a positive deviation from the asymptotic behaviour (see Fig. 4(a)), mainly in the case of the initial $\mathrm{Ag}-\mathrm{TiO}_{2}$ sample which indicates a significant interfacial electron density inhomogeneity, i.e. surface roughness. The calculated values are SR $=2.45$ and $1.25 \mathrm{~nm}$ in the case of Ag$\mathrm{TiO}_{2}$ and $40 \% \mathrm{Ag}-\mathrm{TiO}_{2} / 60 \% \mathrm{~S}$ samples, respectively (see Eq. $\mathrm{E}(6)$ in Supplementary Information): the surface roughness of the samples did not decreased significantly during the addition of sulfur. Because the surface roughness is a crucial factor of the superhy-

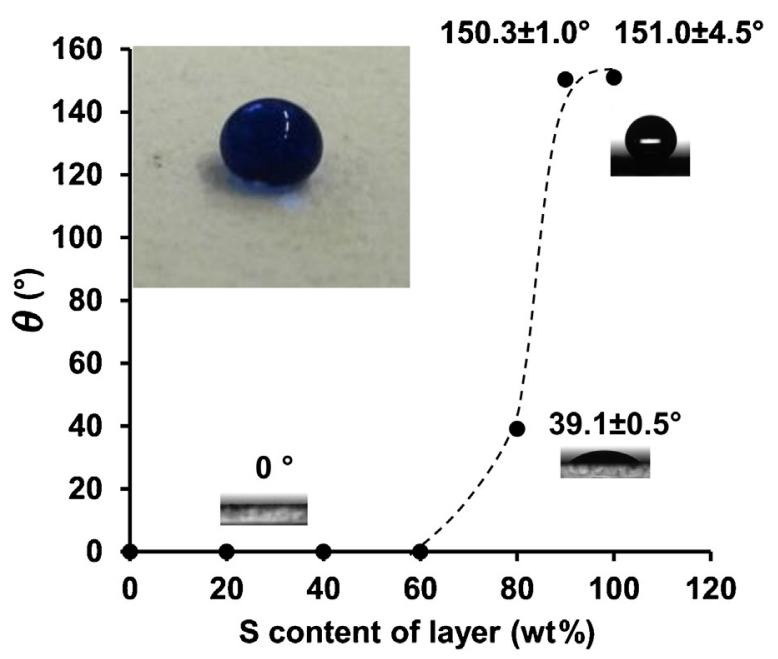

Fig. 5. Apparent static water contact angles on $\mathrm{Ag}-\mathrm{TiO}_{2} / \mathrm{S}$ composite surfaces as a function of S-content. The photograph illustrates the water-repellent behaviour of $90 \mathrm{wt} \%$ S-containing composite powder film with water drop coloured with methylene blue dye.

drophobic coatings, a more detailed description of the composites structure can be found in Supplementary Information (Figs. S1-S3).

\subsection{Wetting and morphological properties and surface free energy determination}

The above presented results indicated that the presence of the sulfur nanoparticles changed the wetting and structural properties of the $\mathrm{Ag}-\mathrm{TiO}_{2}$, which was also evidenced by contact angle measurements (Fig. 5).

As the sulfur content increases, the composite-covered surfaces (spray-coated, with $1 \pm 0.5 \mathrm{mg} / \mathrm{cm}^{2}$ specific layer mass) changed their wetting character from superhydrophilic $\left(\theta \approx 0^{\circ}\right)$ to superhydrophobic $\left(\theta>150^{\circ}\right)$. This sulfur induced superhydrophobic character of the composite layer also can be seen on the inserted photograph in Fig. 5. The water drops coloured with methylene blue a)

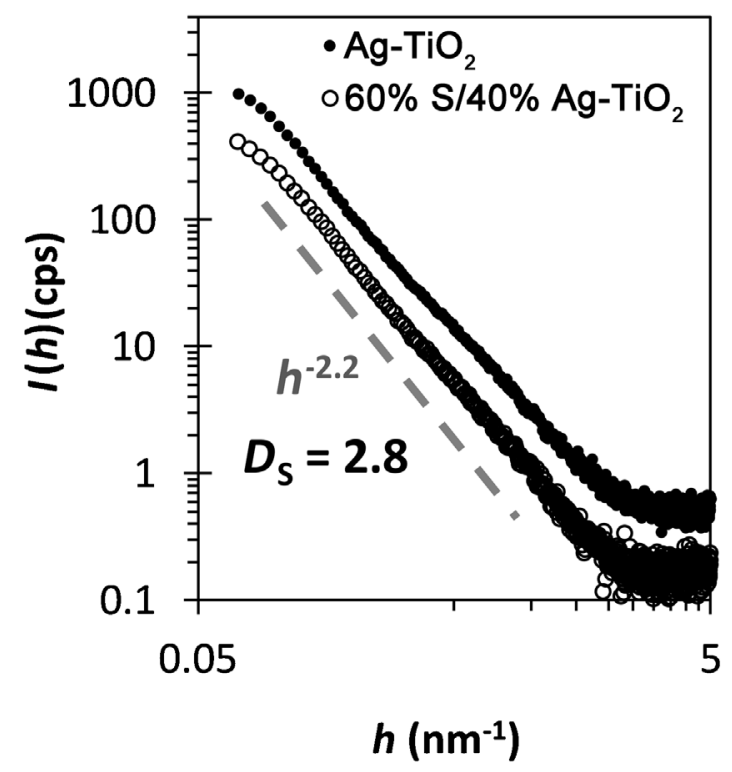

b)

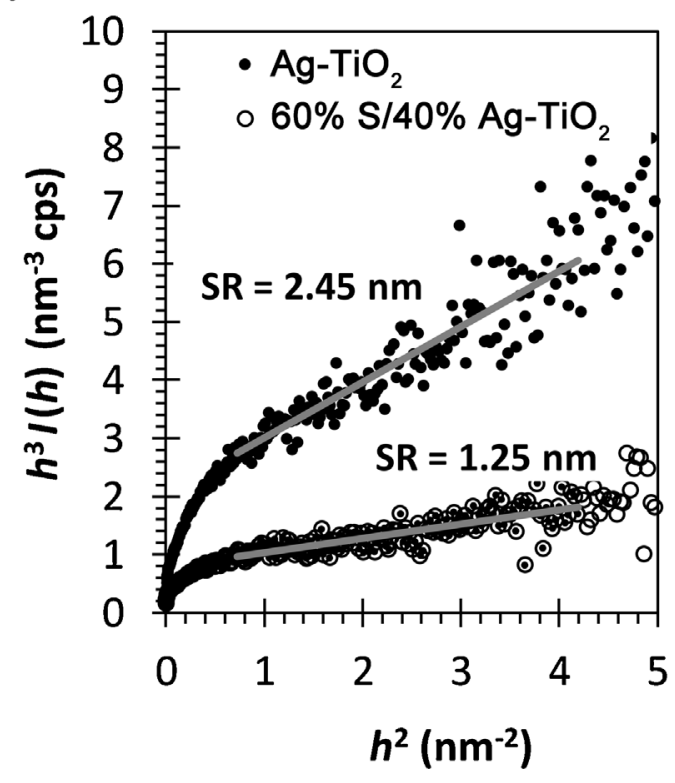

Fig. 4. $I(h)$ vs. $h(\mathrm{a})$ and $h^{3} I(h)$ vs. $h^{2}$ (b) plots of the scattering curves of $\mathrm{Ag}-\mathrm{TiO}_{2}$ and $\mathrm{Ag}-\mathrm{TiO}_{2} / \mathrm{S}$ powder samples with 60 wt\% $\mathrm{S}$-content. 


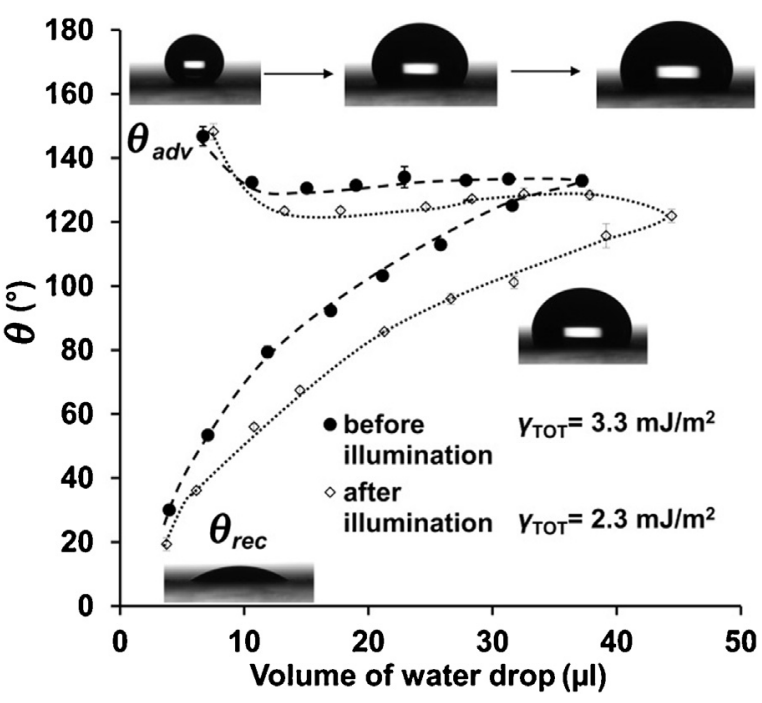

Fig. 6. Evolution of advancing and receding contact angles on spray-coated $90 \mathrm{wt} \%$ sulfur-hydrophobized photoreactive $\mathrm{Ag}$ - $\mathrm{TiO}_{2}$ layers, as well as the determined total apparent surface free energy $\left(\gamma_{\mathrm{s}}{ }^{\text {tot }}\right)$ values before and after $180 \mathrm{~min}$ blue LED light illumination $(\lambda=405 \mathrm{~nm})$.

dye were rolled down from the surface of the superhydrophobic composite film.

Advancing and receding contact angles were also measured on the superhydrophobic film with $90 \mathrm{wt} \% \mathrm{~S}$-content, according to the protocol of Drelich [13]. The data was analysed according to Eq. (1) of Chibowsky [19] to obtain the corresponding total surface free energy values.

Fig. 6 shows a large contact angle hysteresis $\left(\theta_{\mathrm{adv}}=146.8^{\circ} \pm 3.0^{\circ}\right.$ $\left.\rightarrow \theta_{\text {rec }}=30.1^{\circ} \pm 0.6^{\circ}\right)$, indicating the Wenzel-type roughness characteristics of the surface [19]. However, this superhydrophobic nature with high contact angle and low surface energy was durable and resistance for irradiation, because after illuminating the sample with $\lambda_{\max }=405 \mathrm{~nm}$ blue LED-light for $180 \mathrm{~min}$, the $\theta_{\text {rec }}$ values showed a moderate decrease $\left(\theta_{\mathrm{rec}}=19.4^{\circ} \pm 2.0^{\circ}\right)$, while the $\theta_{\mathrm{adv}}$ val-

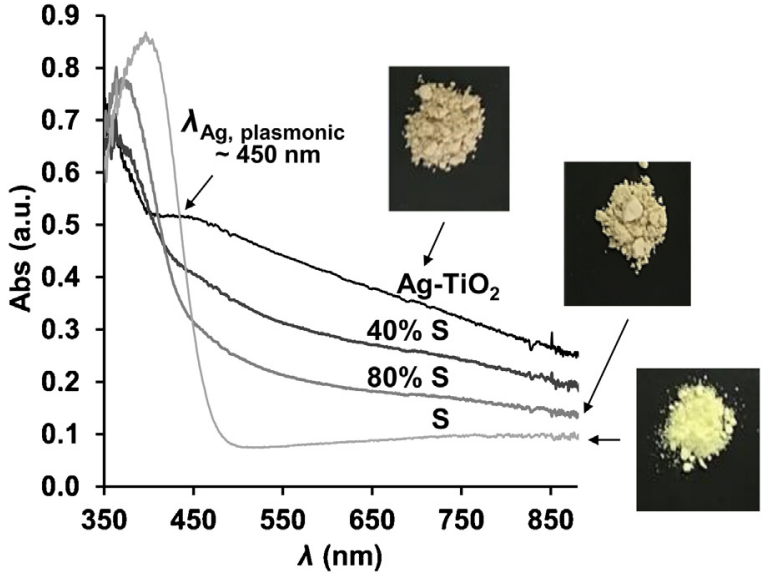

Fig. 8. UV-VIS diffuse-reflectance spectra and photographs of $\mathrm{Ag}_{-} \mathrm{TiO}_{2}, \mathrm{~S}$ nanoparticles and $\mathrm{Ag}-\mathrm{TiO}_{2} / \mathrm{S}$ composites with the corresponding S-content (given in wt\%).

ues remained almost the same $\left(148.2^{\circ} \pm 2.5^{\circ}\right)$, and the calculated total surface free-energy values of the surfaces decreased upon illumination from $3.3 \mathrm{~mJ} / \mathrm{m}^{2}$ to $2.3 \mathrm{~mJ} / \mathrm{m}^{2}$.

As evidenced by SEM-images and EDX-measurements, the surface of the spray-coated layers of $90 \mathrm{wt} \%$ sulfur-hydrophized $\mathrm{Ag}-\mathrm{TiO}_{2}$ photocatalyst has cauliflower-like aggregates (Fig. 7) with relatively homogenous and even distribution of the two components. The presence of both components on the surface of the rough composite film is advantageous because the sulfur nanoparticles are responsible for the superhydrophobic nature, while the presence of free photocatalyst particles ensures the photocatalytic activity. This means that photocatalytic reactions could take place all over the prepared surfaces.

\subsection{Optical and photocatalytic properties}

The optical properties of the hydrophobized photocatalyst samples were slightly changed upon sulfur-addition, which can be seen in the UV-VIS diffuse-reflectance spectra on Fig. 8. The absorption

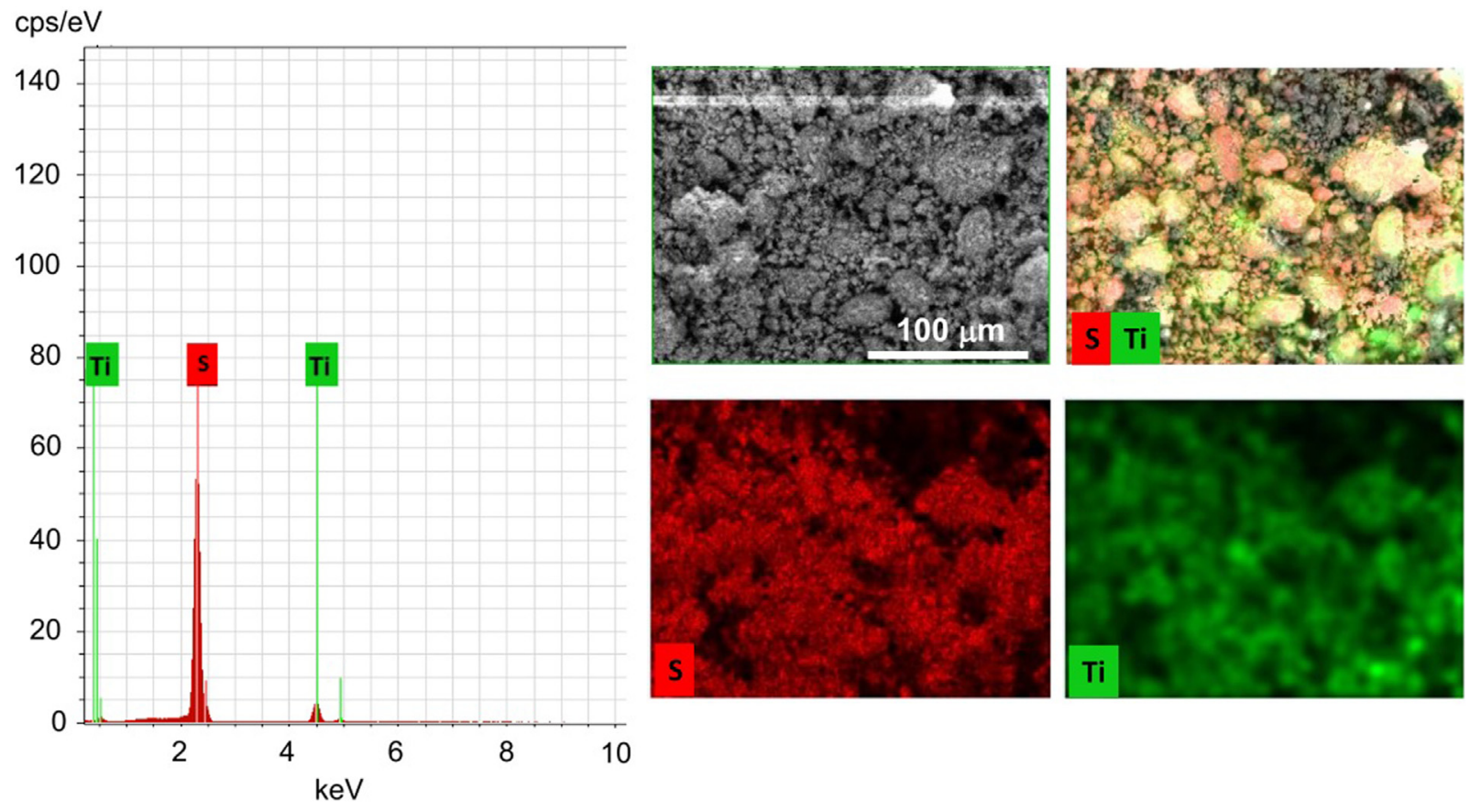

Fig. 7. SEM micrographs and EDX-mapping of $\mathrm{Ag}-\mathrm{TiO}_{2} / \mathrm{S}$ superhydrophobic surfaces with 90 wt\% S-content. 
a)

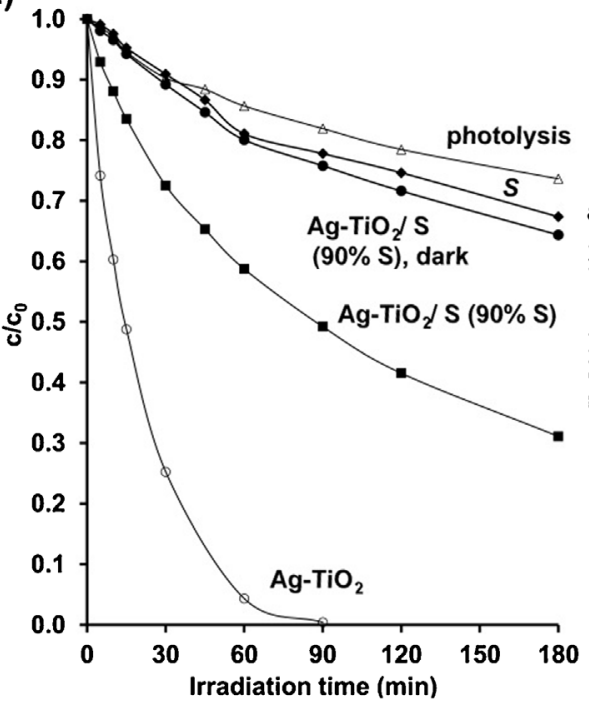

b)

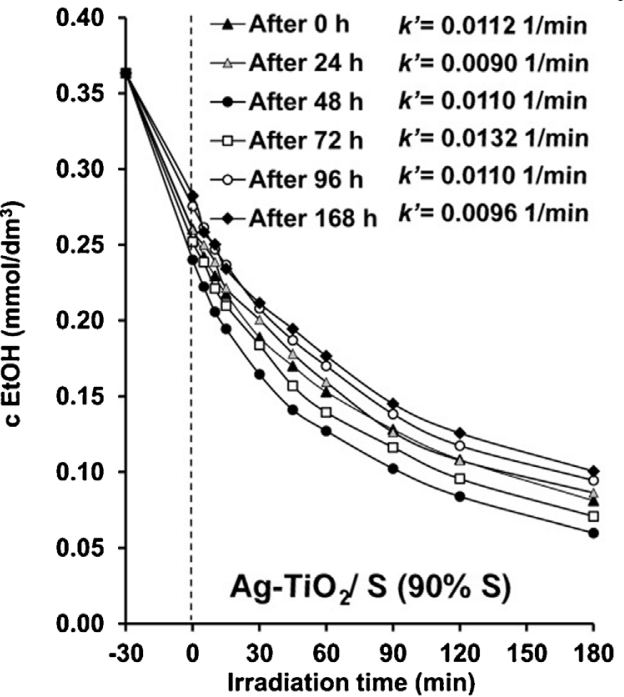

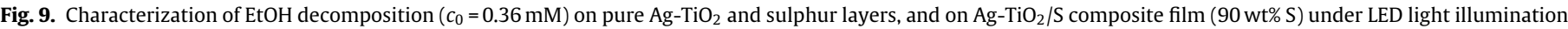

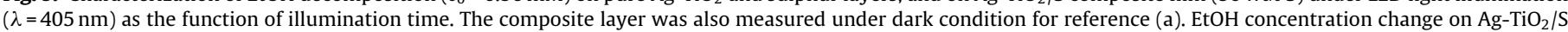
film after $0,24,48,72,96$ and $168 \mathrm{~h}$ continuous LED light irradiation (b).

wavelength maxima gradually increase from 349 to $401 \mathrm{~nm}$ as the sulfur content is increased from 0 to $100 \mathrm{wt} \%$, while the initial pale brown colour of the $\mathrm{Ag}-\mathrm{TiO}_{2}$ gradually turned to yellow. The plasmonic absorbance peak at around $450 \mathrm{~nm}$ is due to the presence of AgNPs on $\mathrm{TiO}_{2}$ photocatalyst particles as demonstrated in our previous papers [4,5]. In this plasmonic photocatalyst, visible light is harvested because the surface plasmon resonance, attributed to the AgNPs, while the metal-semiconductor interface takes part in the separation of the generated holes and electrons [4].

It was also presented earlier that the used blue LED light source with $405 \mathrm{~nm}$ emission maximum is sufficient for excitation of the $\mathrm{Ag}-\mathrm{TiO}_{2}$ catalyst $[31,32]$. In this work the photocatalytic efficiency of the sulfur hydrophobized composite photocatalyst layers were studied using the same blue LED lamp and ethanol as model volatile organic compound (Fig. 9(a)). As a result of 90 min illumination, the pure $\mathrm{Ag}-\mathrm{TiO}_{2}$ photocatalyst film decomposed approximately the whole amount $\left(c_{0}=0.36 \mathrm{mM}\right)$ of the $\mathrm{EtOH}$, while the photocatalytic effectiveness of the sulfur containing superhydrophobic layer were lower, but it was shown significant photocatalytic activity compared to the reference sample (photolysis without photocatalyst and $\mathrm{S} / \mathrm{Ag}-\mathrm{TiO}_{2}$ sample in dark condition). This decreased photocatalytic activity is because of the lower $\mathrm{Ag}-\mathrm{TiO}_{2}$ photocatalyst content (only $10 \%$ in $90 \%$ non-photoreactive sulfur) but according to the wetting (Figs. 5 and 6) and photocatalytic results (Fig. 9), it can be clearly stated that at a composition of $90 \%$ sulfur and $10 \mathrm{wt} \% \mathrm{Ag}-\mathrm{TiO}_{2}$ photocatalyst the composite layer was shown not only superhydrophobic but also photocatalytic properties. In order to prove the long-term photoactivity of the composite sample, the EtOH concentration change was measured occasionally on $\mathrm{S} / \mathrm{Ag}-\mathrm{TiO}_{2}$ film after $0,24,48,72,96$ and $168 \mathrm{~h}$ continuous LED light irradiation. The obtained results in Fig. 9(b) represent that the initial $\left(c_{0}=0.36 \mathrm{mM}\right)$ concentration of EtOH was decreased to $0.26 \pm 0.015 \mathrm{mM}$ after $30 \mathrm{~min}$ adsorption time on the surface of $25 \mathrm{~cm}^{2}$ composite film with $5 \mathrm{mg} / \mathrm{cm}^{2}$ specific surface mass. After this adsorption equilibrium the EtOH concentration was continuously decreased during the LED light irradiation and the calculated $k$ 'values $(0.0090-0.01321 / \mathrm{min})$ indicate the almost unchanged photocatalytic activity of the composite during the studied time interval.

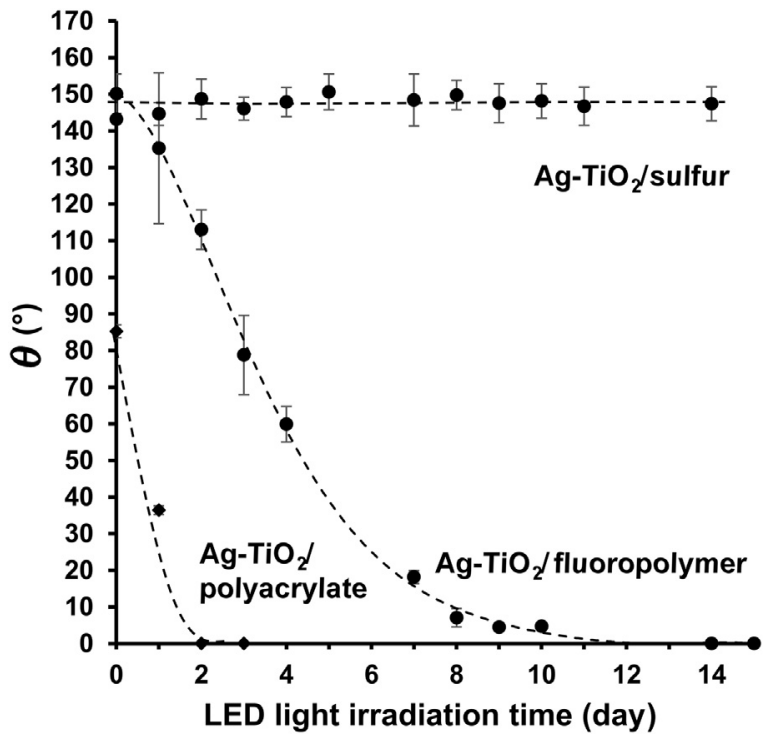

Fig. 10. Effect of long-term UV-A (blue LED) irradiation on the wetting properties of the organic polymer-based and inorganic sulfur-containing superhydrophobic composite coatings.

In our previous work it was reported that this dual superhydrophobic and photocatalytic properties of the spray coated composites surfaces could be very useful in various applications, however, the undesired self-photodegradation of the earlier used organic (fluoro)polymer binder could hinder the application of this coating materials [4,33]. In this work, the hydrophobization of the $\mathrm{Ag}-\mathrm{TiO}_{2}$ photocatalyst particles was achieved by the application of inorganic sulfur in order to avoid its self-photodegradation. Fig. 10 shows that the measured contact angle values of the sulfur hydrophobized $\mathrm{AgTiO}_{2}$ was unchanged $\left(\sim 150^{\circ}\right)$ during the studied time interval (14 days of continuous LED light irradiation).

Two polymer based composites were chosen as reference: the $\mathrm{Ag}-\mathrm{TiO}_{2} /$ polyacrylate hybrid layer contains poly(ethylacrylate-co-methyl methacrylate) copolymer [4], while the 
polymer component of the $\mathrm{Ag}-\mathrm{TiO}_{2} /$ fluoropolymer composite was poly(perfluorodecyl acrylate) fluoropolymer [31,32,34]. As a results, the initial $\theta$ of the $\mathrm{Ag}-\mathrm{TiO}_{2} /$ polyacrylate hybrid layer was $85.2^{\circ} \pm 1.75^{\circ}$ which was drastically changed to $0^{\circ}$ just after two days irradiation. The effect was slower in the case of the more resistance fluoropolymer based binder with initial superhydrophobic $\left(\theta=151.1^{\circ} \pm 5.4^{\circ}\right)$ properties. These decreasing $\theta$ values are the results of the previously reported partial polymer photodegradation: the illumination changes in the ration of catalyst/polymer composition as the photocatalyst particles became uncoated [35]. This resulted in higher catalyst availability on the surface region. However, the sulfur hydrophobized photoreactive thin composite film shows durable hydrophobicity during the irradiation.

Finally the mechanical properties of the prepared $\mathrm{S} / \mathrm{Ag}-\mathrm{TiO}_{2}$ film were also investigated and compared to the fluoropolymer based superhydrophobic thin film. The adhesive tape test was used to get information about the adhesion of the layers [4]. The tape was applied on the coating surface and subsequently was removed by a quick pull. As indicate in Fig. S4, minor amount of superhydrophobic layer could be removed by the tape from both surfaces. However, this mechanical damage caused that both the fluoropolymer and sulfur based layers were lost their superhydrophobicity (see inserted contact angle values) after the peeling. This vulnerability of superhydrophobic surfaces is well known in the literature and it originated from the rough surface and porous structure of these lotus-like coatings $[36,37]$. However, in our previous paper we also presented that the nature inspired self-healing ability of the artificial coatings could solve this problem, if we can achieve that the damaged surface protrusions can regenerate itself [32]. In the future, we try to implement this self-assembled mechanism (air humidity catalysed syneresis of the hydrophobic dodecyltrichlorosilane molecules) into the sulfur containing superhydrophobic layer in order to get thin film with quick superhydrophobic recovery.

\section{Conclusions}

To satisfy the needs for superhydrophobic coatings with increased durability, sulfur also can be applied as an inert, nonphotodegradable and insoluble inorganic hydrophobizing agent. In this paper we presented a simple method to utilize sulfur in the area of functional surfaces: bifunctional, superhydrophobic and photocatalytic coatings were prepared of $90 \mathrm{wt} \%(d \leq 40 \mathrm{~nm})$ sulfur-nanoparticles and $10 \mathrm{wt} \%(d \approx 25 \mathrm{~nm})$ visible light-active $\mathrm{Ag}-\mathrm{TiO}_{2}$ photocatalyst. The surface fixation of sulfur on $\mathrm{Ag}-$ $\mathrm{TiO}_{2}$ particles occurred through electrostatic interaction and although no chemical bond was identified between the components, the initially superhydrophylic photocatalyst nanoparticles $\left(\theta \approx 0.0^{\circ}\right)$ became superhydrophobic $\left(\theta=150.3^{\circ} \pm 1.0^{\circ}\right)$ upon forming composite particles. As evidenced by EDX-SEM, the extreme wetting properties are attributed to the multiscale surface roughness: the spray-coated layers have cauliflower-like aggregates with relatively homogenous distribution of the two components.

The photocatalytic activity of the sulfur containing superhydrophobic layer was lower than that of the pure $\mathrm{Ag}-\mathrm{TiO}_{2}$ film (degraded $100 \%$ of $c_{0}=0.36 \mathrm{M} \mathrm{EtOH}$ in $180 \mathrm{~min}$ ), still showing significant photoreactivity (photocatalytc effectiveness: $68.9 \%$ ) compared to the reference sample (photolysis without photocatalyst; effectiveness: $26.4 \%$ ). This decreased photocatalytic activity is because the lower photocatalyst content (only $10 \%$ ) but accord- ing to the wetting and photocatalytic results it can be clearly stated that at optimal composition the layer was shown not only superhydrophobic but also photocatalytic properties.

As a main result, in contrast to the tested polymer-type hydrophobizing agents, sulfur nanoparticles did not photodegrade in the time interval of 14 days of continuous LED-light illumination $(\lambda=405 \mathrm{~nm})$, so the hydrophobized photocatalyst layers kept their wetting properties $\left(\theta \approx 150^{\circ}\right)$. In conclusion, as the expensive fluoropolymer binders can be replaced with sulfur in self-cleaning superhydrophobic surfaces.

\section{Acknowledgements}

This work was financially supported by the Hungarian Scientific Research Fund (OTKA) K 116323, PD 116224 and the project of GINOP-2.3.2-15-2016-00013, the UNKP-18-4 New National Excellence Program of the Ministry of Human Capacities and by the János Bolyai Research Scholarship of the Hungarian Academy of Sciences and the Ministry of Human Capacities, Hungary (No. 20391-3/2018/FEKUSTRAT).

\section{Appendix A. Supplementary data}

Supplementary material related to this article can be found, in the online version, at doi:https://doi.org/10.1016/j.jmst.2019.04. 046 .

\section{References}

[1] A. Fujishima, T.N. Rao, D.A. Tryk, J. Photochem. Photobiol. C Photochem. Rev. 1 (2000) 1-21.

[2] T. Boningari, S.N.R. Inturi, M. Suidan, P.G. Smirniotis, J. Mater. Sci. Technol. 34 (2018) 1494-1502

[3] W. Liu, Y. Xu, W. Zhou, X. Zhang, X. Cheng, H. Zhao, S. Gao, L. Huo, J. Mater. Sci. Technol. 33 (2017) 39-46.

[4] Á. Veres, J. Ménesi, Á. Juhász, O. Berkesi, N. Ábrahám, G. Bohus, A. Oszkó, G. Pótári, N. Buzás, L. Janovák, I. Dékány, Colloid Polym. Sci. 292 (2014) 207-217.

[5] Á. Veres, T. Rica, L. Janovák, M. Dömök, N. Buzás, V. Zöllmer, T. Seemann, A. Richardt, I. Dékány, Catal. Today 181 (2012) 156-162.

[6] J. Xiong, M.Z. Ghori, B. Herkel, T. Strunskus, U. Schürmann, L. Kienle, F. Faupel, Acta Mater. 74 (2014) 1-8.

[7] Á. Deák, L. Janovák, E. Csapó, D. Ungor, I. Pálinkó, S. Puskás, T. Ördög, T. Ricza, I. Dékány, Appl. Surf. Sci. 389 (2016) 294-302.

[8] F. Zhang, C. Zhang, L. Song, R. Zeng, S. Li, H. Cui, J. Mater. Sci. Technol. 31 (2015) 1139-1143.

[9] I. Dékány, L.G. Nagy, J. Colloid Interface Sci. 147 (1991) 119-128.

[10] M. Bleszynski, M. Kumosa, Compos. Sci. Technol. 164 (2018) 74-81.

[11] K. Chen, W. Gou, Xu Le, Y. Zhao, Compos. Sci. Technol. 156 (2018) 177-185.

[12] Z. Caia, L. Shena, X. Wang, Q. Guo, Compos. Sci. Technol. 164 (2018) 238-247.

[13] J. Drelich, Surf. Innov. 1 (2013) 248-254.

[14] P. Devendar, G.F. Yang, Top. Curr. Chem. (Cham.) 375 (6) (2017) 82-89.

[15] M. Ellis, D. Ferree, R. Funt, L. Madden, Plant Dis. 82 (1998) 428-439.

[16] J. Barkauskas, Res. Bull. 42 (2007) 1732-1741.

[17] Sr. Choudhury, S. Roy, A. Goswami, S. Basu, J. Antimicrob, Chemother. 67 (2012) 1134-1137

[18] W. Zheng, Y.W. Liu, X.G. Hu, C.F. Zhang, Electrochim. Acta 51 (2006) 1330-1340.

[19] E. Chibowski, Adv. Colloid Interface Sci. 103 (2003) 149-172.

[20] U. Eduok, O. Faye, J. Szpunar, Prog. Org. Coat. 111 (2017) 124-163.

[21] A. Gutbier, Z. Anorg, Allergy Chem. 152 (1926) 163.

[22] P.P. von Weimarn, Kolloidchem. Beihefte 22 (1926) 38

[23] M. Raffo, Kolloid-Z 2 (1908) 358.

[24] R. Steudel, Top. Curr. Chem. 230 (2003) 153-166.

[25] T. Preocanin, N. Kállay, Croat. Chem. Acta 79 (2006) 95-106

[26] U. Balachandran, N.G. Eror, J. Solid State Chem. 42 (1982) 276-282.

[27] B. Meyer, Chem. Rev. 76 (1976) 367-388.

[28] M. Ritz, L. Vaculíková, J. Kupková, E. Plevová, L. Bartonová, Vibrat. Spec. 84 (2016) 7-15

[29] A. Caron, J. Donohue, J. Phys. Chem. 64 (1960) 1767-1768.

[30] A.G. Pinkus, J.S. Kim, J.L. McAtee, C.B. Concilio, J. X-Ray, Am. Chem. Soc. 81 (1959) 2652-2654

[31] L. Mérai, Á. Deák, D. Sebök, E. Csapó, T.S. Kolumbán, B. Hopp, I. Dékány, L. Janovak, Express Polym. Lett. 12 (2018) 1061-1071. 
[32] L. Mérai, N. Varga, Á. Deák, D. Sebők, I. Szenti, Á. Kukovecz, Z. Kónya, I. Dékány, L. Janovák, Catal. Today 328 (2019) 85-90.

[33] L. Janovák, Á. Dernovics, L. Mérai, Á. Deák, D. Sebők, E. Csapó, A. Varga, I. Dékány C. Janáky Chem. Commun. 54 (2018) 650-653.

[34] Á. Deák, L. Janovák, E. Csapó, D. Ungor, I. Pálinkó, S. Puskás, T. Ördög, T. Ricza, I. Dékány, Appl. Surf. Sci. 389 (2016) 294-302.
[35] L. Janovák, Á. Deák, Sz.P. Tallósy, D. Sebök, E. Csapó, K. Bohinc, A. Abram, I. Pálinkó, I. Dékány, Surf. Coat. Technol. 326 (2017) 316-326.

[36] H. Xu, C.R. Crick, R.J. Poole, J. Mater. Chem. A 6 (2018) $4458-4465$.

[37] T. Verho, C. Bower, P. Andrew, S. Franssila, O. Ikkala, R.H.A. Ras, Adv. Mater. 23 (2011) 673-678. 